\title{
SANS Study of Lysozyme vs. BSA Protein Adsorption on Silica Nanoparticles
}

\author{
Sugam Kumar ${ }^{1}$, V.K. Aswal ${ }^{1 *}$ and J. Kohlbrecher ${ }^{2}$ \\ ${ }^{1}$ Solid State Physics Division, Bhabha Atomic Research Centre, Mumbai 400 085, INDIA \\ ${ }^{2}$ Laboratory for Neutron Scattering, Paul Scherrer Institute, CH-5232 PSI Villigen, Switzerland \\ "Email:vkaswal@barc.gov.in
}

\begin{abstract}
Lysozyme (M.W. $14.7 \mathrm{kD}$ ) and BSA (M.W. $66.7 \mathrm{kD}$ ) are two most commonly studied model proteins in literature. Lysozyme (cationic) and BSA (anionic) are oppositely charged at $\mathrm{pH} 7$ and their interaction with anionic silica nanoparticles has been studied using small-angle neutron scattering (SANS). Measurements were carried out on fixed 1 wt $\%$ concentration of nanoparticles and varying concentration of protein in the range 0.5 to $2 \mathrm{wt} \%$. It is found that both the proteins adsorb on the nanoparticles where strong interaction of lysozyme leads to the aggregation of nanoparticles but the system remains stable with BSA. Adsorption increases with protein concentration and has been found much larger for lysozyme.
\end{abstract}

Keywords: Protein, Nanoparticles, Adsorption, Aggregation, SANS.

PACS: 81.07.-b, 87.14.Df, 83.85.Hf

\section{INTRODUCTION}

Nanoparticles have wide range of potential applications in the area of nanobiotechnology and nanomedicine because they have sizes comparable to that of living cells and can access to and operate within the cell [1]. Interfacing of biological molecules like proteins to nanoparticles and understanding of their interactions are the prerequisite of applications such as drug delivery and biosensors. The nanoparticle and proteins can interact through various interactions such as covalent bonding, electrostatic forces, and hydrogen bonding etc. depending on the system of interest. It is expected that when the two components are oppositely charged, strong interaction leads to the immediate adsorption of protein molecules on nanoparticle surface and such adsorption diminishes if the two components are likely charged [2]. However, the same is not the case with proteins as their charge distribution (site-specific interaction) plays an important role in their adsorption on the nanoparticles [3]. Herein, we have studied the adsorption of different sized lysozyme and BSA protein at $\mathrm{pH} 7$ where they are oppositely charged on silica nanoparticles using smallangle neutron scattering (SANS). SANS with the possibility to vary contrast is a useful technique to study such multi-component systems [4].

\section{EXPERIMENTAL}

Colloidal suspension of anionic silica nanoparticles (Ludox HS40) and proteins (lysozyme and BSA) were purchased from Sigma-Aldrich and Fluka, respectively. Samples were prepared by dissolving weighted amount of nanoparticle and protein in $20 \mathrm{mM}$ phosphate buffer at $\mathrm{pH} 7$ prepared in $\mathrm{D}_{2} \mathrm{O}$. Small-angle neutron scattering experiments were performed at SANS-I facility, Swiss Spallation Neutron Source SINQ, Paul Scherrer Institut, Switzerland [5]. Data were collected at two sample-to-detector distances 2 and $8 \mathrm{~m}$ to cover a wave vector transfer $(Q)$ range of 0.008 to $0.3 \AA^{-1}$. All the measurements were carried out for fixed $1 \mathrm{wt} \%$ concentration of silica nanoparticles and varying the concentration of protein in the range 0.5 to $2 \mathrm{wt} \%$. The data were corrected and normalized to absolute scale using standard procedure.

\section{SANS ANALYSIS}

In SANS experiments, one measures the coherent differential scattering cross-section per unit volume $(d \Sigma / d \Omega)$ as a function of $Q$, which can be written as [4]

$$
\frac{d \Sigma}{d \Omega}(Q)=n V^{2} \rho_{p}-\rho_{s}{ }^{2} P(Q) S(Q)+B
$$


where $n$ is the number density and $V$ is particle volume. $\rho_{p}$ and $\rho_{\mathrm{s}}$ are scattering length densities of particles and solvent, respectively. $P(Q)$ is intraparticle structure factor and $S(Q)$ is interparticle structure factor. $B$ is a constant term representing incoherent background.

\section{RESULTS AND DISCUSSION}

Fig. 1 shows the SANS data of $1 \mathrm{wt} \%$ lysozyme with 1 wt $\%$ silica nanoparticles along with pure components. The scattering profile of interaction of lysozyme with nanoparticles is quite different from that of lysozyme or nanoparticles. It is also different from that of the addition of the pure components as shown in the inset of Fig. 1. The absence of any correlation peak in the SANS data of lysozyme and nanoparticle allow these data to be fitted as dilute system $(S(Q) \sim 1)$. The spherical silica nanoparticles have the mean size of $16.4 \mathrm{~nm}$ with polydispersity 0.2 whereas lysozyme protein is ellipsoidal having dimensions $4.4 \mathrm{~nm} \times 3.0 \mathrm{~nm} \times 3.0 \mathrm{~nm}$. The buildup of large scattering intensity with linear dependence on $\log$-log scale in the low $Q$ region suggests the particle aggregation as a result of strong interaction of lysozyme with silica nanoparticles [6]. The protein adsorption is fitted with the core-shell structure incorporated in $P(Q)$ whereas the particle aggregation is modeled with $S(Q)$ that of mass fractal. The fractal dimension of particle aggregation is found to be 2.4.

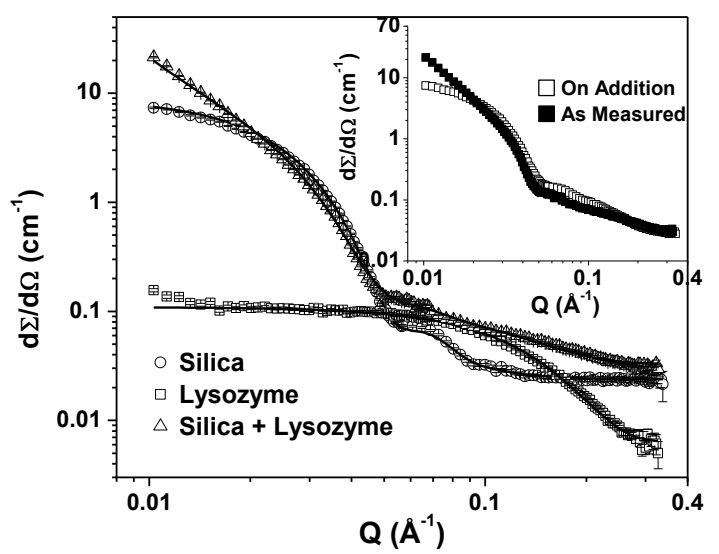

FIGURE 1. SANS data of $1 \mathrm{wt} \%$ Lysozyme with $1 \mathrm{wt} \%$ HS40 silica nanoparticles along with respective pure components. Inset shows the comparison of addition of data for pure components with that experimentally measured.

SANS data of $1 \mathrm{wt} \%$ BSA with 1 wt $\%$ silica nanoparticles along with pure components are shown in Fig. 2. BSA protein is ellipsoidal having dimensions $15.8 \mathrm{~nm} \times 4.6 \mathrm{~nm} \times 4.6 \mathrm{~nm}$. All the data for BSA show similar features to that of lysozyme except that the scattering corresponding to the particle aggregation is not seen. The data are fitted with $P(Q)$ that of the core-shell and $S(Q)$ to that of the dilute system. The adsorption of BSA (22) is found to be much less than that for lysozyme (81) for $1 \mathrm{wt} \%$ of protein.

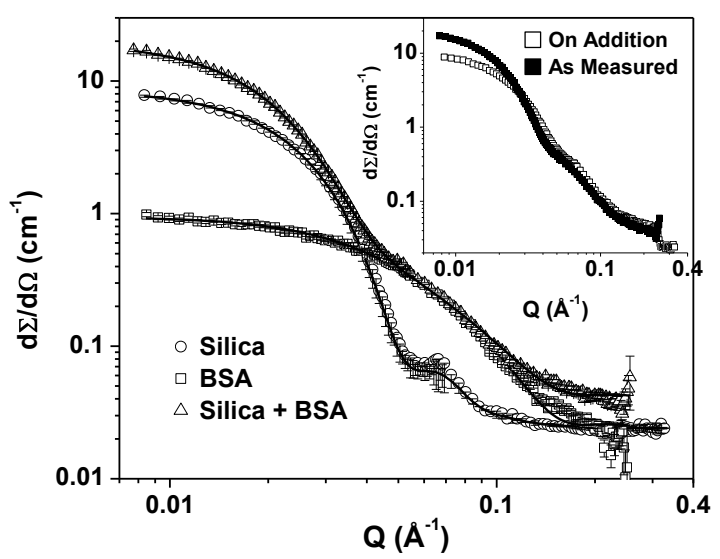

FIGURE 2. SANS data of $1 \mathrm{wt} \%$ BSA with $1 \mathrm{wt} \%$ HS40 silica nanoparticles along with respective pure components. Inset shows the comparison of addition of data for pure components with that experimentally measured.

TABLE 1. Fitted parameters for Protein adsorption on silica nanoparticle.

\begin{tabular}{ccc}
\hline $\begin{array}{c}\text { Protein } \\
\text { concentration } \\
\text { (wt } \%)\end{array}$ & $\begin{array}{c}\text { Adsorbed lysozyme } \\
\text { molecules per } \\
\text { particle }\end{array}$ & $\begin{array}{c}\text { Adsorbed BSA } \\
\text { molecules per } \\
\text { particle }\end{array}$ \\
\hline 0.5 & 53 & 11 \\
1.0 & 81 & 22 \\
2.0 & 90 & 42 \\
\hline
\end{tabular}

Table 1 gives the variation of protein adsorption on silica nanoparticle as a function of protein concentration. The number of adsorbed protein increases for both the proteins and has been found to be much larger for lysozyme. The ratio of protein adsorption of lysozyme to BSA decreases with the protein concentration and this could be because of the saturation effect [6].

\section{REFERENCES}

1. I. Lynch and K. A Dawson, Nano Today 3, 40 (2008).

2. K. Rezwan, A. R. Studart, J. Vö1rö1s, and L. J. Gauckler J. Phys. Chem. B 109, 14469 (2005)

3. K. Rezwan, L.P. Meier, M. Rezwan, J. Vö1rö1s, M. Textor and L. J. Gauckler Langmuir 20, 10055 (2004)

4. Sugam Kumar and V. K. Aswal J. Phys.: Condens. Matter 23035101 (2011).

5. J. Kohlbrecher and W. Wagner, J. Appl. Cryst. 33, 804 (2000)

6. Sugam Kumar, V.K. Aswal and J. Kohlbrecher, Langmuir 27, 10167 (2011) 